\title{
Co-occurrence of mycotoxins in Spanish barley: A statistical overview
}

María Ibáñez-Vea ${ }^{1}$, Elena González-Peñas ${ }^{1}$, Elena Lizarraga ${ }^{1}$, Adela López de Cerain $^{2}$

\author{
${ }^{1}$ Department of Organic and Pharmaceutical Chemistry \\ ${ }^{2}$ Department of Nutrition, Food Sciences, Physiology and Toxicology \\ Faculty of Pharmacy, C.I.F.A., University of Navarra \\ Irunlarrea 1, 31008, Pamplona, Navarra, Spain
}

*Corresponding author:

Elena González-Peñas PhD: Tel.: +34 948 425653. Fax: +34 948425652.

E-mail: mgpenas@unav.es

E-mail addresses of all coauthors: mivea@alumni.unav.es (María Ibáñez-Vea PhD);

elizarraga@unav.es (Elena Lizarraga PhD); acerain@unav.es (Adela López de Cerain $\mathrm{PhD})$. 


\begin{abstract}
In this paper, a statistical overview of the simultaneous presence of 14 mycotoxins in Spanish barley is presented. The co-occurrence of more than two mycotoxins has been observed in $95 \%$ of the samples. From a descriptive standpoint, the results show that the most common combinations were AFB1, OTA and DON (29\%), and AFB1, OTA, DON and ZEA (26\%), although other combinations could also occur. However, the statistical study determined that other associations had a higher impact or importance. Four factors or combinations of variables could explain the $63 \%$ of variability found for the samples. These factors were the associations of: type-B trichothecenes and DAS; type-A trichothecenes and NIV; AFB1, AFG1 and ZEA; and AFB2 and AFG2. The toxin-producing fungi and environmental conditions have been essential in explaining the associations between the toxins.
\end{abstract}

Keywords: mycotoxins, barley, statistical study, factor analysis. 


\section{INTRODUCTION}

The co-occurrence of mycotoxins in foodstuffs and feed can be common because a toxigenic fungus can produce more than one mycotoxin (see table 1), and a foodstuff can be colonized by several fungi either while growing in the field or when it is in storage or during transport. In addition, the processed products are generally composed of various raw materials which may increase the mycotoxin risk (Fink-Gremmels, 2006). Bad practices during harvest and inadequate conditions during drying, handling, packaging, storage and transport may contribute to fungal growth and an increased risk of mycotoxin production (Bhat, Rai and Karim, 2010).

The importance of the co-occurrence of mycotoxins lies in the changes that may occur in the combined toxicity with respect to the toxicity of single mycotoxins. In addition, the existence of relationships in mycotoxin occurrence allows us to assume that the presence of one mycotoxin may indicate the presence of another one. Risk assessment studies usually take into account toxicity and occurrence data of individual mycotoxins. However, it is very important to have reliable data regarding simultaneous presence of toxins in foodstuffs and feed in order to make a better risk assessment for human and animal health. To the best of the author's knowledge, no factor analysis has been carried out in the study of mycotoxin co-occurrence or in the study regarding the factor that could influence their simultaneous presence in raw materials. 
The technical limitations and the chemical diversity of toxins have hindered the study of co-occurrence of mycotoxins in foodstuffs. In fact, there are very few studies in the reference literature that evaluate the simultaneous presence of mycotoxins of different fungi origin and families.

In Spain, very few studies have been carried out which assess the simultaneous presence of mycotoxins in cereal grains, and particularly in barley, despite being the crop with the largest percentage of arable land (52\% in 2007). However, in recently published papers by our research group, mycotoxin co-occurrence in 123 Spanish barley samples has been demonstrated: aflatoxins B1, B2, G1 and G2 (AFB1, AFB2, AFG1 and AFG2, respectively), ochratoxin A (OTA) and zearalenone (ZEA) (Ibáñez-Vea, GonzálezPeñas, Lizarraga and López de Cerain, 2012a); and eight type-A and type-B trichothecenes: deoxynivalenol (DON), nivalenol (NIV), 3-acetyldeoxynivalenol (3-ADON), 15-acetyldeoxynivalenol (15-ADON), fusarenon-X (FUS-X), T-2 and HT-2 toxins and diacetoxyscirpenol (DAS) (Ibáñez-Vea, Lizarraga, González-Peñas and López de Cerain, 2012b).

The aim of this paper is to carry out a statistical study with the results previously obtained about the presence of 14 mycotoxins in barley samples (AFB1, AFB2, AFG1, AFG2, OTA, ZEA, DON, NIV, 3-ADON, 15-ADON, FUS-X, T-2 and HT-2 and DAS), in order to determine whether or not a relationship exists in the simultaneous presence of different mycotoxins and if this association, if statistically significant, may be attributed to a specific factor, e.g. the same producing fungi or climate conditions favoring different fungi species growth. 


\section{MATERIAL AND METHODS}

\subsection{Barley samples}

One hundred and twenty-three barley samples were collected from the 2007 and 2008 harvests. Sample characteristics, and their collection are described in Ibáñez-Vea et al. (2012a). Briefly, most of the samples were provided by national factories dedicated to the production of foodstuffs and feed, after applying their own sampling procedures for cereal quality control; other samples were collected from agricultural cooperatives. All samples are from Navarra, a northern region of Spain that is characterized by its climatic diversity, divided into four climatic areas. The climate in the northwestern area is temperate maritime warm, with an annual rainfall of $1100-2500 \mathrm{~L} / \mathrm{m}^{2}$ and an average annual temperature of $8.5-14.5^{\circ} \mathrm{C}$. The annual mean rainfall in the northern area ranges between 700 and $2200 \mathrm{~L} / \mathrm{m}^{2}$, and the mean temperature ranges between 7 and $15^{\circ} \mathrm{C}$. The central area of Navarra is characterized by a Mediterranean climate (annual mean rainfall: $450-1100 \mathrm{~L} / \mathrm{m}^{2}$; annual mean temperature: $11-14^{\circ} \mathrm{C}$ ). The southern area is the driest part of Navarra (annual mean rainfall lower than $500 \mathrm{~L} / \mathrm{m}^{2}$ ) and the average annual temperature is $14^{\circ} \mathrm{C}$. The moisture content of the samples was less than $15 \%$ upon arrival at the laboratory and the samples were then stored at $4^{\circ} \mathrm{C}$ until their analysis.

\subsection{Mycotoxin analysis}

Samples were analyzed using two in-house validated methods. One of them was an UHPLC-FLD method developed for the determination of aflatoxins (AFB1, AFG1, AFB2 and AFG2), OTA and ZEA (Ibáñez-Vea, Corcuera, Remiro, Murillo-Arbizu, González-Peñas and Lizarraga, 2011a). The second one was a GC-MS method 
developed for the quantification of eight type-A and type-B trichothecenes (DON, 3-ADON, 15-ADON, NIV, FUS-X, T-2, HT-2 and DAS) (Ibáñez-Vea, Lizarraga and González-Peñas, 2012b).

\subsection{Statistical analysis}

The statistical analysis of the data was performed with the SPSS program 15.0. The study has taken into account the mycotoxin levels between the LOD and LOQ; in the case of a value lower than the LOD, half of the LOD value was used (making mandatory the use of nonparametric methods). The samples having levels higher than the LOD have been considered to be positive samples.

Correlation between the levels of two toxins has been verified with the Spearman's Rank Correlation test. The statistical significance was determined using a probability value of 0.05 or 0.01 .

Factor analysis was developed for evaluating the possible associations between different toxins. The KMO value (Kaiser-Meyer-Olkin), a measure of the sampling adequacy, and the Bartlett's Test of Sphericity were evaluated in order to determine whether or not the factor analysis was suitable.

\section{RESULTS AND DISCUSSION}

\subsection{Co-occurrence of mycotoxins}

Incidence and concentration levels of mycotoxins were previously published (see Ibáñez-Vea et al. (2012a) for AFB1, AFG1, AFB2, AFG2, OTA and ZEA; and IbáñezVea et al. (2012b) for type-A and type-B trichothecenes (DON, 3-ADON, 15-ADON, NIV, FUS-X, T-2, HT-2 and DAS)). 
Taking into account incidence and level of the 14 mycotoxins in the 123 barley samples, the results show that ninety-five percent of the samples presented detectable levels of three or more mycotoxins (see figure 1). The most common combinations were AFB1, OTA and DON (29\%), and AFB1, OTA, DON and ZEA (26\%). In addition, the appearance of DON together with its acetylated compounds (15-ADON: 57\%; 3ADON: $28 \%$ ) and ZEA (37\%) was also frequent. When DON and 15-ADON occurred, ZEA or OTA was present in approximately half of the samples (43 and $57 \%$, respectively). The samples were also contaminated with DON and type-A trichothecenes, although in a lower proportion, with the co-occurrence of DON and HT2 being observed in $22 \%$ of the samples. The simultaneous presence of DON and HT-2 together with ZEA or OTA took place in 30 and $74 \%$ of the samples, respectively.

\subsection{Factor analysis}

The Spearman's Rank Correlation test has shown that relationships exist between the toxins that were studied. All of the toxins have presented relationships with, at least, another one, except in the case of AFB1 and FUS-X. In table 2, correlation coefficient significant at the 0.05 and 0.01 levels are identified with asterisks. In view of these results, it has been considered adequate to evaluate the possible relationship between the toxins by means of factor analysis. As a result of the low correlation between OTA and FUS-X with other toxins, OTA and FUS-X have not been taken into account in the factor analysis. 
Before carrying out factorial analysis the KMO value (Kaiser-Meyer-Olkin), a measurement of the sampling adequacy, and the Bartlett's Test of Sphericity have been evaluated in order to determine if the factorial analysis was suitable. The KMO value, which compares the observed correlation coefficients to partial correlation coefficients, was 0.700 . This result indicates that the degree of common variance among the 12 variables is middling, and that factor analysis is appropriate for this data and produces distinct and reliable factors. Lower values for KMO suggest problems with the sampling, with the degree of common variance among the items being mediocre (KMO from 0.60 to 0.69 ) o miserable (KMO from 0.50 to 0.59 ). In addition, the Bartlett's Test was highly significant $(\mathrm{p}<0.001)$ and therefore, there are some relationships between the studied variables, and the factorial analysis is appropriate.

The principal component analysis and the Varimax rotation indicated that $63 \%$ of the variability of the results might be justified by several factors related with 4 combinations of mycotoxins. Each one of these factors is responsible for the 23, 19, 11 and $10 \%$ of variability found for the samples, respectively. This has been supported by the scree plot that showed that four factors on the curve are above the eigenvalue of 1 (see figure 2), so at least four factors are needed to justify the results.

The rotated component matrix is displayed in table 3. This is a matrix of the factor loadings for each variable onto each factor. The factor analysis has grouped the 12 variables in 4 components. The mycotoxin loads on factor 1 all appear to relate to toxins produced mainly by Fusarium graminearum and F. culmorum fungi, although DAS cannot be explained by this association due to the fact that it is produced mainly by $F$. sporotrichioides. The second component links the type-A trichothecenes amongst each 
other and to a lesser extent, it links the type-A trichothecenes to NIV. This fact suggests that T-2, HT-2 and DAS might be produced by F. sporotrichioides, but also by powdery F. poae, which is also able to produce NIV. The third factor has grouped AFB1, AFG1 and ZEA, but this association is not due to the toxin-producing fungi, because aflatoxins are produced by Aspergillus, and ZEA is produced by Fusarium. Finally, the last component is formed by AFB2 and AFG2, also produced by the Aspergillus species.

These associations can be explained not only by the mycotoxin-producing fungi but also by the environmental factors which favor the growth of the fungi and the production of the mycotoxins. In the case of DAS, its link with type-B trichothecenes is strange because these two groups of mycotoxins are produced by different fungi, but this fact may be due to climatic conditions. As in the case of DON, the highest value found for DAS was in a sample in the southern Navarra, the area with the majority of DON contamination, although the incidence was higher in central Navarra. This suggests that climatic conditions characteristic of this central area may favor the growth of the DASproducing fungi, but the warm climate of southern Navarra favors the production of DAS. The association of nivalenol with type-A trichothecenes is easy to explain if it is taken into account the fact that powdery $F$. poae, one of the main type-A trichothecene producers, can also synthesize NIV. Moreover, the maximum level found was observed in the central region of Navarra although the occurrence was less than in southern Navarra. This indicates the same hypothesis formulated for DAS, in which it is possible that climatic conditions favorable for the growth of the fungi, are not favorable for the production of the toxin. Therefore, NIV may present higher incidence in the south as a result of better growth of the fungi, but the highest levels found in the central Navarra 
zone suggest that the climatic conditions of this region are more favorable for the production of this toxin.

The lack of a relationship between AFB1 and AFB2 could denote the little importance that A. flavus have with regard to the production of these toxins, implying that their production may be mainly due to $A$. parasiticus, which produce aflatoxins B and G. On the one hand, the association between AFB1 and AFG1 and between AFB2 and AFG2 suggest the different environmental requirements for the production of these toxins and the prevalence of a pathway of synthesis in different conditions. On the other hand, the association between AFB1 and AFG1 and ZEA could indicate that AFB1 and AFG1 contamination may be generated or initiated in the field in the same conditions as ZEA, or that the presence of ZEA in the grains could enable contamination with AFB1 and AFG1.

Despite the fact that DON and ZEA are produced by the same fungi, this survey did not find a high correlation between the two toxins, thereby suggesting that the two toxins are not co-contaminants. Two main chemotypes of $F$. graminearum $/ G$. zeae which produce either DON and its acetylated compounds (3-ADON or 15-ADON) or NIV and FUS-X, with ZEA as a possible metabolite of both chemotypes, have been described (Miller, Grennhalgh, Wang and Lu, 1991). The kinetics of DON and ZEA formation could condition the levels of both toxins because previously published research had observed that the concentration of DON in corn cobs inoculated in the field with $F$. graminearum increased rapidly whereas the production of ZEA started later and increased at a significantly lower rate (Muller, Reimann, Schumacher and Schwadorf, 1997). If this fact also occurs in barley, it could explain the higher content of DON compared to ZEA. Other researchers have analyzed the two toxins in barley, with the 
incidence and levels of ZEA being lower than those of DON (Edwards, 2009; Mankeviciene, Butkuté, Dabkevicius and Suproniene, 2007). However, to the best of the author's knowledge, the relationship between these two toxins has not been reported, despite having observed that they occur together.

\section{CONCLUSIONS}

Results obtained in the study of the co-occurrence of 14 mycotoxins in 123 barley samples have been analyzed together in order to determine the possible relationships between the different toxins that are present. The co-occurrence of more than two mycotoxins has been observed in $95 \%$ of the samples. From a descriptive standpoint, results showed that the most common combinations were AFB1, OTA and DON (29\%), and AFB1, OTA, DON and ZEA (26\%), although other combinations could also occur. However, the statistical study determined that other associations had a higher impact or importance. The factor analysis shows that four factors or combinations of variables could explain $63 \%$ of the variability in the samples. These factors were the associations of: type-B trichothecenes and DAS; type-A trichothecenes and NIV; AFB1, AFG1 and ZEA; and AFB2 and AFG2. These associations might be explained by mycotoxinproducing fungi in each case and also by climatic conditions which favor crop infection.

\section{ACKNOWLEDGEMENTS}

The authors are grateful to the personnel at the participating local grain delivery sites for their cooperation. We wish to extend our gratitude to Ms. Laura Stokes for reviewing the English version of this manuscript and to Dr. García-Granero for reviewing the statistical studies. We thank the Project of Research of University of Navarra (PIUNA Projects) and CAN (Caja Navarra; "Proyectos Tú eliges, tú decides") for the financial 
support received. The authors are grateful to the reviewers, whose comments and suggestions have helped to improve this article. 


\section{BIBLIOGRAPHY}

Bhat, R., R. V. Rai, \& A. A. Karim (2010). Mycotoxins in food and feed: Present status and future concerns. Comprehensive Reviews in Food Science and Food Safety, 9 5781.

Edwards, G. (2009). Fusarium mycotoxin content of UK organic and conventional barley. Food Additives Contaminants: Part A: Chemistry, Analysis, Control, Exposure Risk Assessment, 26(8), 1185-1190.

Fink-Gremmels, J. (2006). Moulds and mycotoxins as undesirable substances in animal feeds, In Barug, D., Bhatnagar, D., van Egmond, H. P., van der Kamp, J. W., van Osenbruggen, W. A., \& Visconti, A., The mycotoxin factbook. Food and feed topics (pp. 37-50). Wageningen Academic Publishers.

Ibáñez-Vea, M., E. González-Peñas, E. Lizarraga, \& A. López de Cerain (2012a). Cooccurrence of aflatoxins, ochratoxin A and zearalenone in barley from a northern region of Spain. Food Chemistry. 132, 35-42.

Ibáñez-Vea, M., L. A. Corcuera, R. Remiro, M. T. Murillo-Arbizu, E. González-Peñas, \& E. Lizarraga (2011a). Validation of a UHPLC-FLD method for the simultaneous quantification of aflatoxins, ochratoxin A and zearalenone in barley. Food Chemistry, 127(1), 351-358.

Ibáñez-Vea, M., E. Lizarraga, \& E. González-Peñas (2011b). Simultaneous determination of type-A and type-B trichothecenes in barley samples by GC-MS. Food Control, 22 1428-1434. 
Ibáñez-Vea, M., E. Lizarraga, E. González-Peñas, \& A. López de Cerain (2012). Cooccurrence of type-A and type-B trichothecenes in barley from a northern region of Spain. Food control, 25, 81-88.

Mankeviciene, A., B. Butkuté, Z. Dabkevicius, \& S. Suproniene (2007). Fusarium mycotoxins in Lithuanian cereals from the 2004-2005 harvests. Annals of Agricultural and Environmental Medicine, 14, 103-107.

Miller, J. D., R. Grennhalgh, Y. Z. Wang, \& M. Lu (1991). Trichothecene chemotypes of 3 Fusarium species. Mycologia, 83(2), 121-130.

Muller, H. M., J. Reimann, U. Schumacher, \& K. Schwadorf (1997). Natural occurrence of Fusarium toxins in barley harvested during five years in an area of southwest Germany. Mycopathologia, 137, 185-192. 


\section{Figure captions}

Figure 1.. Number of mycotoxins in a sample versus percentage of samples in which these mycotoxins co-occur

Figure 2. Scree plot. 
Figures

Figure 1

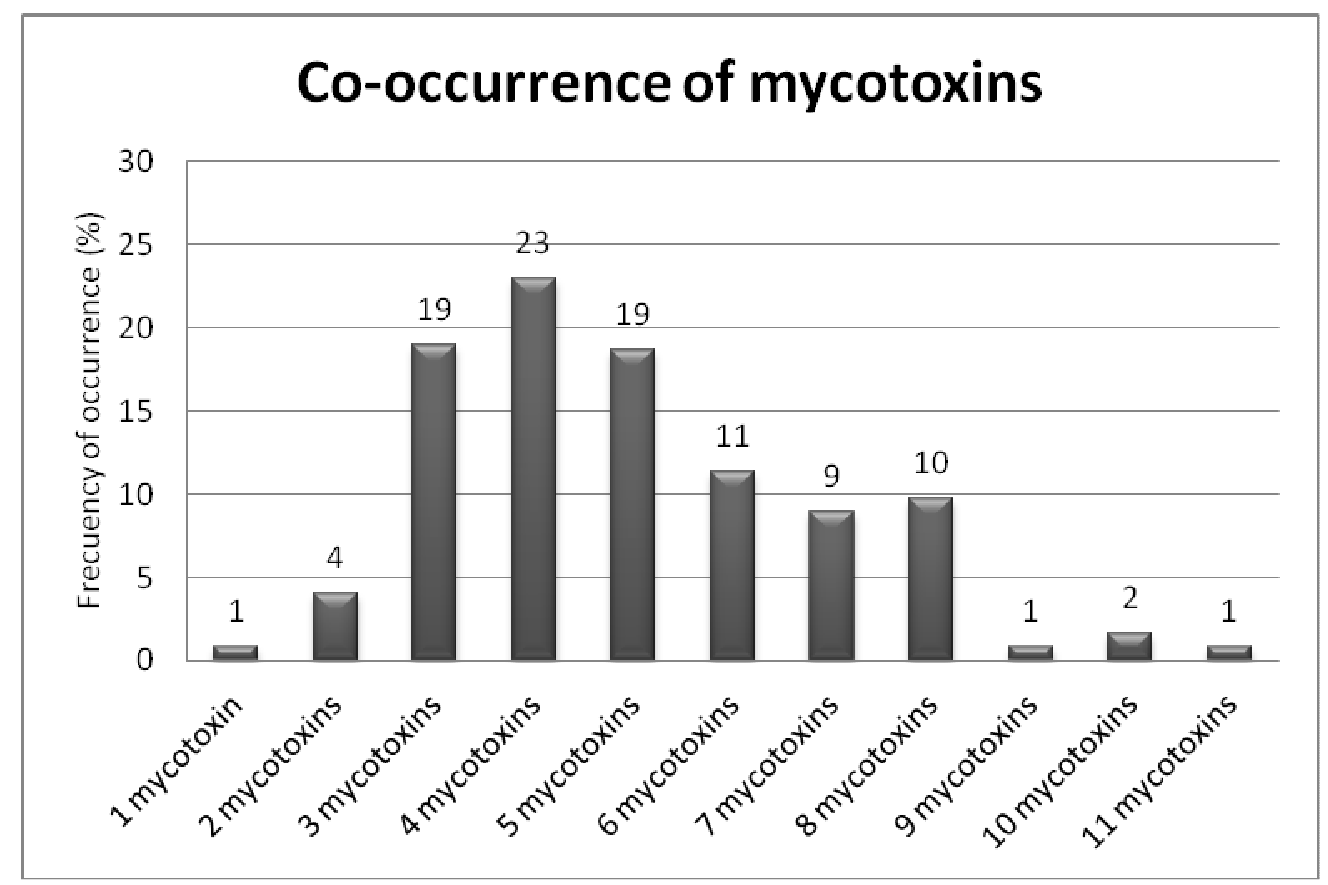


Figure 2






\section{Tables}

Table 1. Fungal species optimal growth conditions and the mycotoxins they may produce.

\begin{tabular}{c|c}
\hline Fungal species & Mycotoxins \\
\hline F. culmorum & DON, 3-ADON, 15-ADON, NIV, FUS-X, ZEA \\
F. graminearum & DON, 15-ADON, NIV, FUS-X, ZEA \\
F. sporotrichioides & T-2, HT-2, NEO, DAS, FUS-X ZEA \\
F. poae & T-2, HT-2, NIV, DAS, FUS-X \\
F. moniliforme & FMs, moniliformin, fusarin C \\
F. oxysporum & Moniliformin, wortmannin, fusaric acid, \\
F. sambucinum & sambutoxin \\
A. flavus & Sambutoxin \\
A. parasiticus & Aflatoxins, cyclopiazonic acid \\
A. ochraceus & Aflatoxins \\
P. verrucosum & Ochratoxins \\
\hline
\end{tabular}


Table 2. Correlation matrix.

\begin{tabular}{|c|c|c|c|c|c|c|c|c|c|c|c|c|c|c|c|}
\hline & & AFG2 & AFG1 & AFB2 & AFB1 & ZEA & OTA & DON & NIV & FUS-X & 15-ADON & 3-ADON & DAS & HT-2 & T-2 \\
\hline \multirow[t]{14}{*}{ Correlation } & AFG2 & & & & & & & & & & & & & & \\
\hline & AFG1 & 0.008 & & & & & & & & & & & & & \\
\hline & AFB2 & $0.189^{*}$ & -0.004 & & & & & & & & & & & & \\
\hline & AFB1 & 0.099 & 0.174 & 0.027 & & & & & & & & & & & \\
\hline & ZEA & $0.213^{*}$ & $0.216^{*}$ & 0.080 & 0.113 & & & & & & & & & & \\
\hline & OTA & -0.141 & 0.021 & $-0.186^{*}$ & 0.027 & $0.199 *$ & & & & & & & & & \\
\hline & DON & 0.001 & -0.045 & 0.204 & 0.162 & 0.141 & -0.041 & & & & & & & & \\
\hline & NIV & 0.001 & -0.010 & 0.040 & 0.163 & 0.066 & 0.080 & $0.290 * *$ & & & & & & & \\
\hline & FUS-X & -0.060 & -0.029 & -0.058 & 0.014 & -0.099 & -0.011 & 0.113 & 0.109 & & & & & & \\
\hline & 15-ADON & 0.052 & -0.085 & 0.146 & 0.219 & 0.152 & 0.012 & $0.758^{* *}$ & $0.450^{* *}$ & 0.044 & & & & & \\
\hline & 3-ADON & -0.037 & 0.041 & 0.181 & 0.121 & 0.197 & -0.011 & $0.723^{* *}$ & $0.256^{* *}$ & $0.214^{*}$ & $0.551^{* *}$ & & & & \\
\hline & DAS & 0.052 & -0.030 & 0.027 & 0.139 & 0.023 & 0.016 & $0.440^{* * *}$ & $0.475^{* *}$ & 0.041 & $0.452^{* * *}$ & $0.274^{* *}$ & & & \\
\hline & HT-2 & -0.033 & 0.049 & -0.024 & -0.035 & -0.111 & 0.160 & 0.143 & $0.373^{* *}$ & 0.075 & 0.175 & 0.066 & $0.491 * *$ & & \\
\hline & $\mathrm{T}-2$ & 0.005 & 0.044 & -0.080 & -0.012 & -0.07 & 0.029 & 0.025 & $0.310^{* * *}$ & -0.042 & 0.070 & 0.045 & $0.305 * *$ & $0.659^{* *}$ & \\
\hline
\end{tabular}

* Correlation is significant at the 0.05 level (bilateral). ** Correlation is significant at the 0.01 level (bilateral). 
Table 3. Rotated component matrix.

\begin{tabular}{l|cccc}
\hline \multirow{2}{*}{} & \multicolumn{4}{|c}{ Component } \\
\cline { 2 - 4 } & 1 & 2 & 3 & 4 \\
\hline DON & 0.908 & & \\
15-ADON & 0.847 & & \\
3-ADON & 0.810 & & \\
HT-2 & & 0.880 & \\
T-2 & & 0.832 & \\
DAS & 0.459 & 0.622 & & \\
NIV & 0.406 & 0.570 & & \\
AFG1 & & & 0.782 & \\
ZEA & & & 0.594 & 0.827 \\
AFB1 & & & 0.575 & 0.656 \\
AFG2 & & & & \\
AFB2 & & & & \\
\hline
\end{tabular}

